\title{
Effect of the GPI anchor of human Thy-1 on antibody recognition and function
}

\author{
John E Bradley', Joy M Chan² and James S Hagood ${ }^{3}$
}

Thymocyte differentiation antigen-1 (Thy-1) is a glycosylphosphatidylinositol (GPI)-linked cell surface glycoprotein expressed on numerous cell types, which regulates signals affecting cell adhesion, migration, differentiation, and survival. In addition, Thy-1 has been detected in the serum, cerebral spinal fluid, wound fluid from venous ulcers, synovial fluid from joints in rheumatoid arthritis, and, more recently, urine. We previously detected Thy- 1 in the conditioned media of cytokine-stimulated lung fibroblasts, suggesting that Thy-1 shedding may be a response to cellular stress. Soluble and membrane-bound forms of Thy-1 from in vivo sources have been shown to be identical in size when deglycosylated, suggesting that soluble Thy-1 is separated from the diacyl glycerol portion of its GPI anchor by hydrolysis within the GPI moiety. For Thy-1- and other GPI-anchored proteins, delipidation induces a stable change in conformation that manifests itself in a change in antibody affinity for soluble forms. Using epitope-tagged recombinant soluble Thy-1, we report that widely available monoclonal antibodies to human Thy- 1 are unable to detect soluble Thy-1 by immunoblotting. We re-evaluated the Thy- 1 that we previously reported in the conditioned media of normal human lung fibroblasts and found it to be entirely insoluble. These findings suggest that most Thy-1 reported in body fluids retains its GPI anchor and may be associated with membrane fragments or vesicles. This phenomenon should be considered in the generation of antibodies and controls for Thy- 1 bioassays. Furthermore, the changes in Thy- 1 conformation with delipidation, beyond affecting antibody affinity, likely affect the ligand affinity and biological function of soluble vs released membraneassociated forms.

Laboratory Investigation (2013) 93, 365-374; doi:10.1038/labinvest.2012.178; published online 28 January 2013

KEYWORDS: antibody recognition; delipidated Thy-1; fibroblast; GPI anchor; recombinant Thy-1

Thy-1 cell surface antigen (also thymocyte differentiation antigen-1, CD90 or Thy-1), a glycosylphosphatidylinositol (GPI)-linked cell surface glycoprotein on stem cells and multiple mature cell types, was originally discovered in an attempt to raise antiserum against leukemia-specific antigens from the $\mathrm{C} 3 \mathrm{H}$ mouse strain in the AKR mouse strain and vice versa. The antibodies were found to label strongly thymoctyes as well as peripheral $\mathrm{T}$ cells. ${ }^{1}$ For this reason, the original designation for the antigen changed from Theta to Thy- $1 .^{2}$ The cell types expressing Thy- 1 in normal and pathological conditions, and the immunological and non-immunological roles of Thy-1 have been reviewed elsewhere. ${ }^{3-5}$

Our lab previously reported that normal human lung fibroblasts (NHLFs) treated with several different proinflammatory cytokines undergo a decrease in the level of cell surface Thy- 1 expression. This decrease coincides with an increase of detectable Thy- 1 in the conditioned media $(\mathrm{CM})$, suggesting the release of Thy- 1 from the cell surface. From these observations, we concluded that Thy- 1 is likely separated (shed) from the diacyl glycerol portion of its GPI anchor by a 'GPIase' hydrolyzing a bond within the GPI moiety. ${ }^{6}$ Others have demonstrated Thy-1 detectable in the serum, wound fluid, urine, and cerebral spinal fluid in a variety of normal and pathological conditions. ${ }^{7-9}$ Taken together, these findings suggested that released Thy-1 may serve as a useful biomarker for certain pathological conditions. In addition, recent studies have shown that a soluble recombinant Thy-1 in which the GPI-attachment signal is replaced by the Fc fragment of human IgG1 (Thy-1-Fc) alters

\footnotetext{
${ }^{1}$ Department of Medicine, Division of Immunology and Rheumatology, University of Alabama at Birmingham, Birmingham, AL, USA; ${ }^{2}$ Department of Pediatrics, University of California-San Diego, La Jolla, CA, USA and ${ }^{3}$ Pediatric Respiratory Medicine, University of California-San Diego and Rady Children's Hospital of San Diego, La Jolla, CA, USA

Correspondence: Dr JS Hagood, MD, Pediatric Respiratory Medicine, University of California-San Diego and Rady Children's Hospital of San Diego, 9500 Gilman Drive, MC 0731, La Jolla, CA 92093-0731, USA.

E-mail: jhagood@ucsd.edu

Received 14 October 2012; accepted 27 November 2012
} 
activation of latent transforming growth factor- $\beta$ and cell phenotype in lung fibroblasts, ${ }^{10}$ indicating that Thy-1 may have a role as a soluble mediator in addition to its function on the cell surface. For these reasons, antibody detection of Thy-1 in biological fluids is important in clinical and research applications.

However, antibody-mediated detection or purification of Thy-1 is known to be problematic for several reasons. Many of the monoclonal antibodies employed to detect Thy- 1 in western blots require that it be prepared under non-reducing (NR) conditions, including the clones OX7, G7, HO-13-4, 5E10, and ASO2. ${ }^{8,11-13}$ For many antibodies that recognize GPI-anchored proteins at the cell surface, their affinity is lost or greatly diminished if that same protein is delipidated. This phenomenon has been reviewed elsewhere. ${ }^{14}$ For Thy-1 in particular, several monoclonal and polyclonal antibodies to mouse Thy-1 have been shown not to react with delipidated, soluble Thy-1 (sThy-1). ${ }^{11,12}$ Although mostly demonstrated for antibodies to mouse Thy-1, a group reported the reactivity of an antiserum raised against membrane-bound human Thy-1 failed to recognize hydrophilic human Thy-1 purified from cerebral spinal fluid. For Thy-1 and other GPIanchored proteins, the general consensus is that delipidation induces a stable change in conformation that manifests itself in a change in antibody affinity. ${ }^{14}$ Antibodies that recognize human Thy-1 with a GPI anchor are predicted to have lower affinity for Thy- 1 if it is delipidated. Therefore, we reasoned that the Thy-1 we detected in fibroblast supernatants and others detected in several body fluids using antibodies to membrane-bound Thy-1 may not be a truly soluble, cleaved form.

The widely used monoclonal antibodies K117 (American Type Culture Collection Number: HB-8553), 5E10 (STEMCELL Technologies; catalog no. (cat. no.) 01437), and AS02 (Millipore; cat. no. CP28) were generated from mice immunized with a human astrocytoma cell line, a human erythroleukemia cell line, and human dermal fibroblasts, respectively. The antigen recognized at the cell surface by K117, 5E10, and AS02 is Thy-1. We performed the following studies to characterize recognition of soluble and GPIanchored forms of Thy-1 by antibodies from these clones.

\section{MATERIALS AND METHODS}

\section{Recombinant Constructs of THY1}

For the expression of wild-type (WT) human Thy-1, the complete cDNA of human THY1 (human gene symbol for Thy-1) (AAH65559.1) was ligated into the mammalian expression vector pcDNA3.1/Zeo $(+)$ (Invitrogen; cat. no. V860-20) with the Kozak sequence $5^{\prime}$-GCCGCC- $3^{\prime 15}$ just upstream of the start codon. The restriction sites EcoRI and NotI were used at the $5^{\prime}$ and $3^{\prime}$ end, respectively (Figure 1a and b). To express mature Thy- 1 with an $\mathrm{N}$-terminal FLAG tag, a coding sequence for the FLAG epitope $e^{16,17}$ was cloned immediately downstream of that of the ER localization signal $^{18}$ and just upstream of the first codon for mature

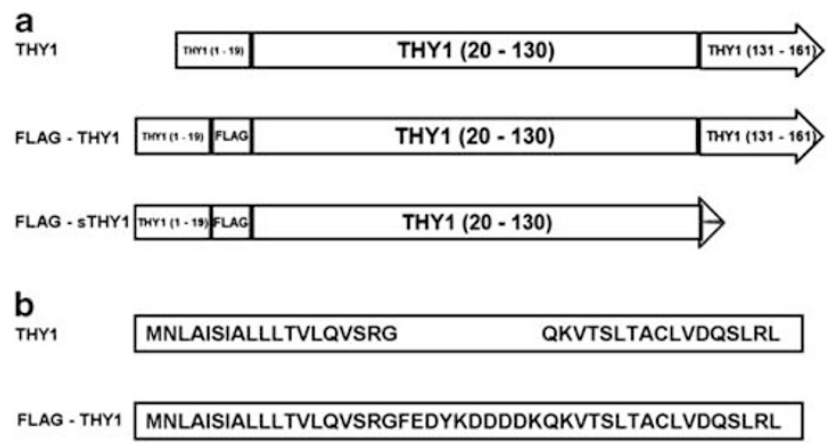

Figure 1 Primary structures of $\mathrm{N}$ terminally tagged recombinant thymocyte differentiation antigen 1 (Thy-1). (a) Full-length diagrams of primary structures for wild-type (WT) Thy- 1 and $\mathrm{N}$ terminally tagged recombinant Thy-1. (b) Alignment of the amino-acid (AA) sequences at the $\mathrm{N}$ terminus of $\mathrm{N}$ terminally tagged recombinant Thy- 1 with $\mathrm{WT}$ Thy- 1 . S, soluble.

Thy-1 (Figure 1a and b). To express Thy-1 with its GPIattachment signal replaced with that of another glycoprotein, coding sequences for the foreign GPI-attachment signal were cloned downstream of a 'hinge' region. This 'hinge' region comprises the six amino acids (AA) downstream of mature Thy-1, EGISLL, changed to GGIGLS as was previously shown to successfully add the transmembrane domains of cluster of differentiation 8 and neural cell adhesion molecule to mouse Thy-1. ${ }^{19}$ Using site-directed mutagenesis, an intermediate construct was made first by changing the sequence just downstream of the codons for mature Thy- 1 to code for GGIG, followed by the BstBI restriction site. Expression vectors containing the cDNA of the GPI-attachment signals for decay-accelerating factor $(D A F)$ and TNF-related apoptosis-inducing ligand receptor 3 (TRAIL-R3) were kindly provided by Daniel F Legler (University of Konstanz, Konstanz, Germany). The cDNA of TRAIL-R3 contains multiple threonine-, alanine-, proline-, and glutamine-rich repeats that present multiple annealing sites for the $5^{\prime}$ cloning primer. To avoid this possibility, the TRAIL-R3-containing expression vector was cut with $P v u I I$ and NotI producing a $115 \mathrm{bp}$ fragment. This fragment was used as the template for PCR. PCR products of TRAIL-R3 and DAF had the restriction sites of BstBI and NotI introduced 24 base pairs upstream of the final codon in the mature protein and after the stop codon, respectively. These PCR products were cloned into the intermediate construct with BstBI and NotI. Finally, the BstBI sites in both were converted to codons for LS by site-directed mutagenesis (Figure $2 \mathrm{a}$ and $\mathrm{b}$ ). To express sThy-1 with a C-terminal histidine tag, an intermediate construct was made introducing half the changes required for six histidines, and then a stop codon to follow the 'hinge' region by site-directed mutagenesis. The intermediate was then used as the template in another round of site-directed mutagenesis to complete the required changes (Figure 2a and b). To express sThy-1 with an N-terminal FLAG tag, the 'hinge' region was introduced followed by a stop codon into 


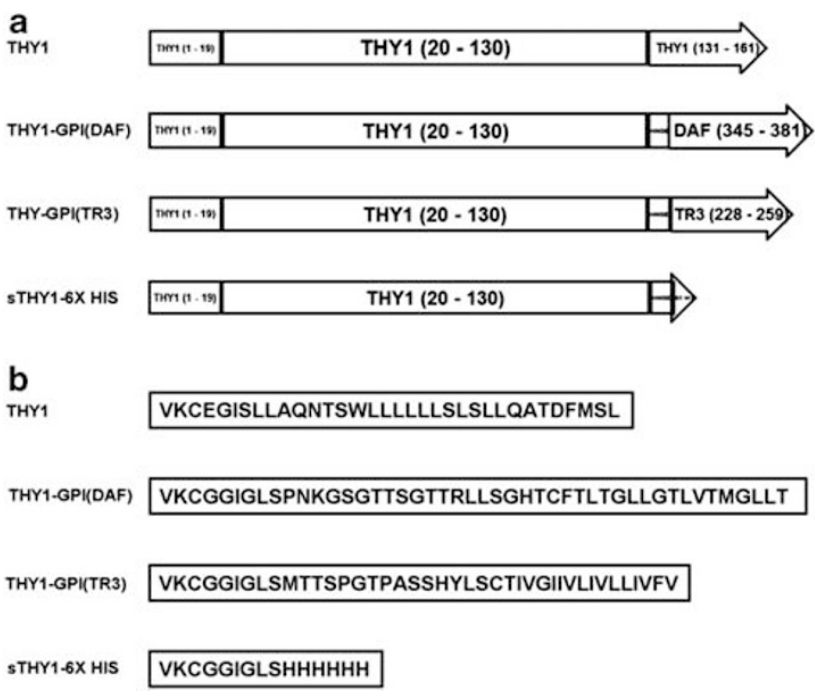

Figure 2 Primary structures of recombinant thymocyte differentiation antigen 1 (Thy-1) with modifications to the $C$ terminus. (a) Full-length diagrams of primary structures for wild-type (WT) Thy-1 and recombinant THY1 with modifications to the $\mathrm{C}$ terminus. (b) Alignment of the aminoacid (AA) sequences at the $C$ terminus of recombinant Thy- 1 with modifications to the $C$ terminus with WT Thy-1. DAF, decay-accelerating factor; GPI, glycosylphosphatidylinositol; $6 \times$ HIS, 6-histidine; s, soluble.

FLAG-THY1 by site-directed mutagenesis (Figure 1a and b). See Figures 1 and 2 for schematics of these constructs.

\section{Cell Culture of RFL-6 and CCL-210}

Rat fetal lung fibroblasts (RFL-6) (American Type Culture Collection) were maintained in F-12K supplemented with $10 \%$ FBS, $1 \%$ penicillin, and $1 \%$ streptomycin with medium exchanged every 2 days. CCL-210 (American Type Culture Collection), NHLF, were maintained in MEM supplemented with $10 \%$ FBS, $1 \%$ penicillin, and $1 \%$ streptomycin, with the medium exchanged every 2 days. Both were passaged using $0.25 \%$ trypsin. Cell extracts (CE) and CM were collected in the following manner. Adherent cells were washed two times with PBS, and then harvested by scraping into ice-cold IP lysis buffer (Pierce; cat. no.87788). Cell lysates were incubated on ice for $5 \mathrm{~min}$ with periodic vortexing, and then centrifuged at $13000 \mathrm{~g}$ for $10 \mathrm{~min}$ at $4{ }^{\circ} \mathrm{C}$. The supernatant containing the $\mathrm{CE}$ was stored at $-80^{\circ} \mathrm{C}$ before use. $\mathrm{CM}$ from adherent cells was cleared of cellular debris by centrifugation at $1200 \mathrm{~g}$ for $5 \mathrm{~min}$ at $4{ }^{\circ} \mathrm{C}$. The supernatant was either immediately concentrated using Amicon's Ultra-2 Centrifugal Filter Unit with a $3 \mathrm{kDa}$ retention membrane, precipitated with methanol, subjected to differential centrifugation, or stored at $-80^{\circ} \mathrm{C}$. For cytokine treatment, cells at $90 \%$ confluency were washed with serum-free media (SFM), and then serum starved in SFM for $24 \mathrm{~h}$, followed by culture in either MEM alone or $20 \mathrm{ng} / \mathrm{ml}$ each TNF $\alpha$ (Gibco; cat. no. PHC3015L) and IL-1 $\beta$ (Gibco; cat. no. PHC815) in MEM for 24 to $48 \mathrm{~h}$.

\section{Stable Expression of Recombinant Thy-1 in RFL-6}

RFL-6 were transfected using Lipofectamine 2000 (Invitrogen; cat. no. 11668) according to the manufacturer's protocol. After $24 \mathrm{~h}$, cells were subcultured in growth media so as to be near $10 \%$ confluency the following day, then washed with SFM, and later cultured in growth media supplemented with $500 \mu \mathrm{g} / \mathrm{ml}$ Zeocin (Invitrogen). Stably transfected cells that were selected after several passages showed no visual indication of cell death.

\section{Deglycosylation of Thy-1 with PNGase F}

CEs or CMs were diluted in water and brought to $1 \%$ SDS with a $10 \%$ stock solution, and then incubated at $100{ }^{\circ} \mathrm{C}$ for $10 \mathrm{~min}$, following the manufacturer's standard protocol for peptide: N-glycosidase F (PNGase F) (New England BioLabs; cat. no. P0704), except for omission of dithiothreitol from the denaturing buffer. An aliquot was taken as the control for Thy-1 before deglycosylation. G7 reaction buffer and NP40 were added to a final concentration of $1 \times$ and $1 \%$, respectively. After adding PNGase F, this reaction mixture was incubated at $37^{\circ} \mathrm{C}$ for $1 \mathrm{~h}$, and then diluted with PBS to the same total protein concentration.

\section{Release of Thy-1 from Cell Surfaces with PI-PLC}

Cells expressing Thy- 1 were cultured in growth media to near $90 \%$ confluency, washed with SFM, and then incubated with $0.1 \mathrm{U} / \mathrm{ml}$ phosphatidylinositol-specific phospholipase C (PI-PLC) (Invitrogen; cat. no. P-6466) in SFM for $24 \mathrm{~h}$.

\section{Analysis of Cell Surface Thy-1 Expression by Flow Cytometry}

Flow cytometry was performed as described previously ${ }^{6}$ using the following antibodies: anti-human Thy- 1 and mouse IgG1 $\kappa$ isotype control conjugated to FITC (BD Pharmingen; cat. nos. 555595 and 555748, respectively).

\section{Western Blot Analysis}

Unless otherwise specified, all samples were prepared in NR SDS loading buffer (50 mM Tris- $\mathrm{HCl}$ (pH 6.8), 2\% SDS, and $10 \%$ glycerol). Sample preparations were electrophoresed in $10 \%$ polyacrylamide gels, and then transferred onto PVDF membranes. Depending on the primary antibody, membranes were either blocked in 5\% milk in TBST or TBST alone. The primary antibodies for detecting the mature Thy- 1 polypeptide, K117, 5E10, and AS02, were diluted to $0.1 \mu \mathrm{g} / \mathrm{ml}$ in TBST. The primary antibodies for detecting the FLAG (Sigma-Aldrich; cat. no. M2 F1804) and 6 histidine $(6 \times$ HIS) epitopes (Rockland; cat. no. 600-401-382) were diluted to $1.0 \mu \mathrm{g} / \mathrm{ml}$ in $5 \%$ milk in TBST. All primary antibodies were incubated with membranes overnight at $4{ }^{\circ} \mathrm{C}$. Antibodies bound to antigen were visualized with secondary antibodies conjugated to horseradish peroxidase in conjunction with a chemiluminescent substrate. The secondary antibodies used were goat anti-mouse $(\mathrm{H}+\mathrm{L})$ (Bio-Rad; cat. no. 172-1011) and goat anti-rabbit $(\mathrm{H}+\mathrm{L}$ ) (Thermo Scientific; cat. no. 
32460) diluted $1 / 200000$ and $1 / 40000$ in blocking buffer, respectively. When required, membranes were stripped with Pierce's restore western blot stripping buffer following the manufacturer's protocol.

\section{Partitioning of CM using Triton X-114}

Triton X-114 (Sigma-Aldrich; cat. no. 93422) was added to concentrated CM to a final concentration of $2 \%$. An aliquot was taken as the control for total Thy- 1 before partitioning. With periodic vortexing throughout, the remainder was kept on ice for $10 \mathrm{~min}$, and then another $10 \mathrm{~min}$ at $37^{\circ} \mathrm{C}$. Following this, the sample was immediately centrifuged at $21000 \mathrm{~g}$ for $10 \mathrm{~min}$ at room temperature. Centrifuging produced a clear partition between the insoluble and soluble phases. Before western blot analysis, volumes were adjusted to represent equal fractions of starting volume of $\mathrm{CM}$.

\section{Differential Centrifugation and Methanol Precipitation of $\mathrm{CM}$}

Two $150 \mu \mathrm{l}$ aliquots were taken from the supernatant of $\mathrm{CM}$ centrifuged at $1200 \mathrm{~g}$ for $5 \mathrm{~min}$ at $4{ }^{\circ} \mathrm{C}$. One aliquot was centrifuged again at $21000 \mathrm{~g}$ for $5 \mathrm{~min}$ at $4^{\circ} \mathrm{C}$. After this, the supernatant was removed. All samples were precipitated by adding $1350 \mu \mathrm{l}$ of methanol, and then leaving them at $-20{ }^{\circ} \mathrm{C}$ overnight. The following day, all were centrifuged at $21000 \mathrm{~g}$ for $30 \mathrm{~min}$ at $4{ }^{\circ} \mathrm{C}$. The supernatant was poured off and the precipitant was allowed to air dry before being resuspended in $35 \mu \mathrm{l}$ of $1 \times$ SDS loading buffer, and then submitted to western blot analysis.

\section{RESULTS}

\section{Anti-Human Thy-1 Monoclonal Antibodies Which React with Thy-1 at the Cell Surface do not Recognize Delipidated Thy-1}

Human Thy-1 from CE is readily detected by western blot in NR conditions using either K117, 5E10, or AS02 monoclonal antibody (Figure $3 \mathrm{a}$ and $\mathrm{b}$ and Supplementary Figure 1). The NHLF cell line CCL-210 was treated with PI-PLC as described in the Materials and methods section. Analysis by flow cytometry showed that Thy-1 was completely removed by PI-PLC (data not shown). However, Thy-1 was not detected in the CM of PI-PLC-treated CCL-210 using the K117 monoclonal antibody, despite being easily detected in CEs diluted to the same volume as the CM (data not shown).

To confirm the presence of Thy-1 released by PI-PLC into $\mathrm{CM}$, a recombinant form was engineered to be expressed at the cell surface with an N-terminal FLAG tag. This was accomplished by introducing the FLAG epitope ${ }^{16,17}$ downstream of the N-terminal ER localization signal ${ }^{18}$ and just upstream of the first residue of mature Thy-1, designated FLAG-THY1 (Figure 1a and b). The size of FLAG-THY1 stably expressed by RLF- 6 relative to WT Thy- 1 is in keeping with the predicted polypeptide mass difference of $\sim 1.3 \mathrm{kDa}$ and with being correctly glycosylated (Figure 3a). CEs from RFL-6 stably expressing FLAG-THY1 and the CM of the same

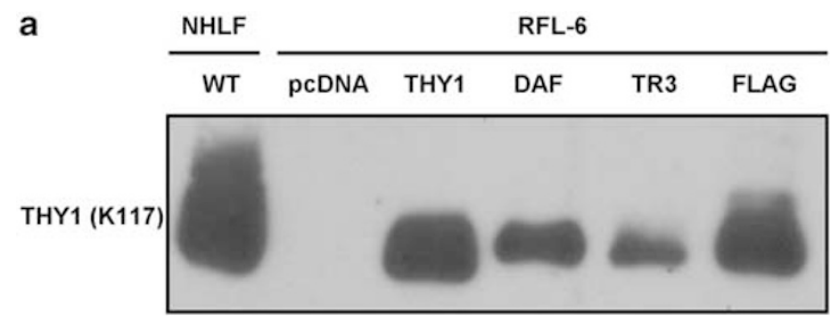

b

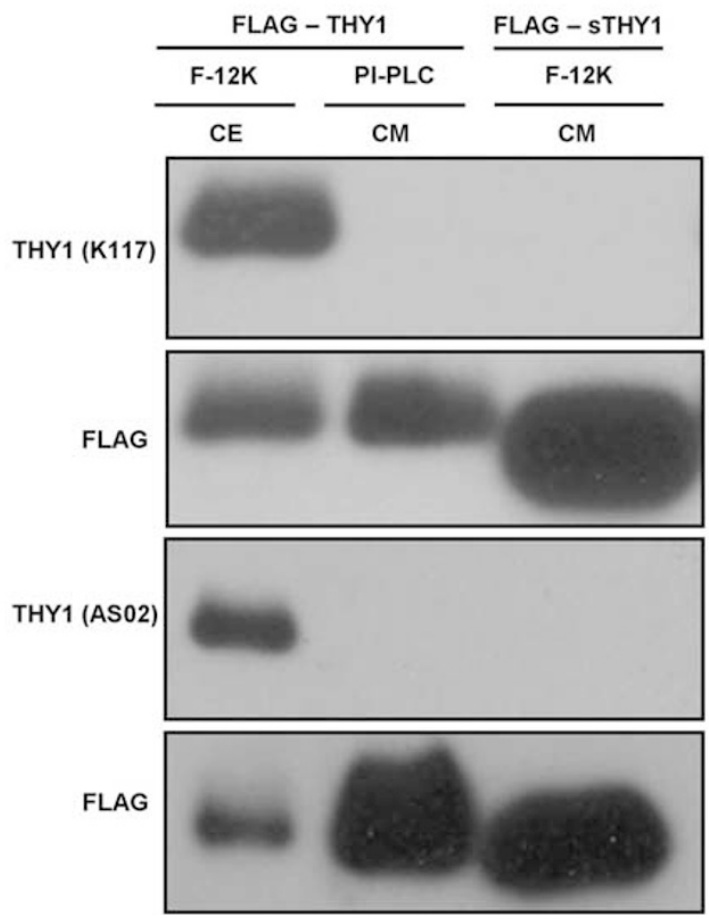

Figure 3 Complete glycosylphosphatidylinositol (GPI) anchor of thymocyte differentiation antigen 1 (Thy-1) required for recognition by monoclonal antibodies raised against membrane-bound form. (a) Mature Thy- 1 detected by western blot in the cell extracts (CEs) of normal human lung fibroblast (NHLF) and rat fetal lung fibroblast (RFL)- 6 stably transfected with pcDNA3.1/Zeo $(+)$ (pcDNA) or pcDNA3.1/Zeo $(+)$ with a recombinant form of Thy-1 cloned in: wild-type (WT) THY1 (THY1), THY1GPI(DAF) (DAF), THY1-GPI(TR3) (TR3), or FLAG-THY1 (FLAG). (b) Mature Thy-1 detected by western blot in CE containing FLAG-Thy-1 or conditioned media (CM) containing phosphatidylinositol-specific phospholipase C (PI-PLC) released FLAG-Thy-1 or FLAG-sThy-1 using K117 and AS02. After stripping the membranes of antibodies for detecting mature Thy-1, FLAG was detected using M2. DAF, decay-accelerating factor; GPI, glycosylphosphatidylinositol; s, soluble.

cell line treated with PI-PLC were compared in western blots with anti-FLAG as the primary antibody (data not shown). Using FLAG band intensity to control for loading of Thy-1, CM from PI-PLC-treated fibroblasts were used in western blots with clone K117 5E10, and AS02 as the primary antibodies. Each western blot showed a band in the lane corresponding to the $\mathrm{CE}$ but not the $\mathrm{CM}$ (Figure $3 \mathrm{~b}$ and Supplementary Figure 1) After stripping the membranes of primary and secondary antibodies for detecting Thy-1, each was re-probed with FLAG antibody. Unlike with clones K117, 5E10, and ASO2, the immunoblot for FLAG revealed a band 
in each lane equal in size. However, the band appearing in the lane in which PI-PLC released FLAG-THY1 was run was equal or greater in intensity as predicted (Figure $3 \mathrm{~b}$ ). Thus, sufficient FLAG-THY1 was present for clones K117, 5E10, and AS02 to detect Thy-1 were it not delipidated.

\section{Monoclonal Antibodies from Clones K117 and 5E10 Recognize Epitopes on Thy-1 Independent of its Glycosylation, but are Abolished Under Reducing Conditions}

Glycosylation of Thy-1, which is exclusively N-linked, can account for more than $50 \%$ of its total mass. Between different cell and tissue types, the carbohydrate moiety composition may vary dramatically. As a consequence, the molecular mass of Thy- 1 ranges from 25 to $37 \mathrm{kDa}$. $712,13,20$ Of the anti-mouse monoclonal antibodies that have affinity for non-delipidated Thy-1, OX7, H140-150, H154-177, ${ }^{12}$ and AS $02^{13}$ have been demonstrated to recognize their respective epitopes independent of glycosylation. Therefore, the epitopes lost with delipidation are likely confined to a region of the Thy-1 polypeptide. The mature Thy- 1 polypeptide has four cysteine residues. As a member of the immunoglobulin superfamily, each cysteine is predicted to form a disulfide bond with one of the other under oxidizing conditions. $^{21}$ Western blots to detect Thy-1 that utilize antibodies from clones $\mathrm{HO}-13-4, \mathrm{G} 7,{ }^{11} \mathrm{OX} 7,{ }^{12} 5 \mathrm{E} 10,{ }^{8}$ or $\mathrm{ASO} 2^{13}$ as the primary are conducted under NR conditions. NR conditions are used with clone K117 as well (Figure 3).

To determine if recognition by clones K117 and 5E10 is contingent on glycosylation or disulfide bonds, both were used as the primary antibodies in western blots of native, reduced, and completely deglycosylated Thy- 1 in CCL-210 extract. PNGase F cleaves between the innermost GlcNAc and asparagine of oligosaccharides from N-linked glycoproteins and was shown to remove completely the glycans from human Thy-1. ${ }^{7}$ With clone K117 (Figure 4) and 5E10 (data not shown), a single band of a size corresponding to fully glycosylated Thy-1 was detected in the lane containing nonPNGase F-treated CE prepared under NR conditions. To circumvent possible diffusion of the reducing agent into adjacent lanes, samples were run in western blots with an empty lane between those with reducing agents and those without (Figure 4). No bands were detected in the lanes containing non-PNGase F-treated cell lysate prepared under reducing conditions. A single band of a size corresponding to completely deglycosylated Thy-1, $\sim 14 \mathrm{kDa}$, was detected in the lane containing PNGase F-treated CE prepared under NR conditions. Moreover, the bands were equal in intensity and relative size (Figure 4). As is the case with clones OX7, H140150, H154-177, and AS02, the epitopes recognized by K117 and 5E10 on human Thy-1, which are lost with delipidation, remain with deglycosylation. In addition, breaking disulfide bonds with reducing agents completely abolishes epitope recognition by these antibodies.

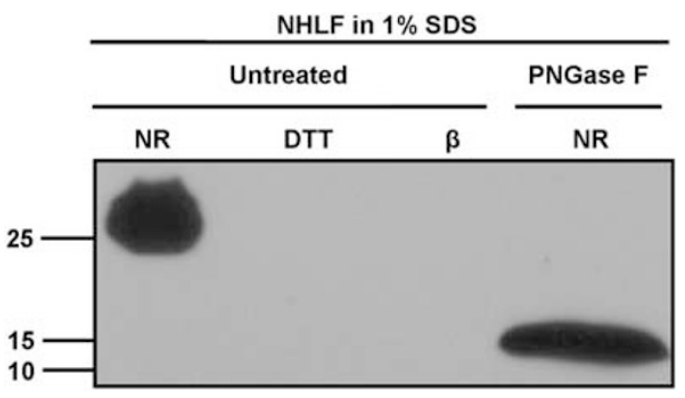

Figure 4 Monoclonal antibodies from clones K117 and 5E10 recognize epitopes on thymocyte differentiation antigen 1 (Thy-1) independent of its glycosylation, but are abolished under reducing conditions. Mature Thy-1 detected by western blot in equal dilutions of untreated or peptide: N-glycosidase F (PNGase F)-treated normal human lung fibroblast (NHLF) extract prepared as indicated under non-reducing (NR) conditions or with $100 \mathrm{mM}$ dithiothreitol (DTT) or $2.5 \% \beta$ mercaptoethanol $(\beta)$. SDS, sodium dodecyl sulfate.

\section{Substitution of the Native GPI Anchor-Attachment Signal of THY1 with the GPI-Attachment Signals of DAF or TRAIL-R3 does not Alter Antibody Reactivity Despite the Presence of an Intervening 15 AA Region}

Recombinant THY1 hybrids were engineered to have GPI anchors attach using the DAF and TRAIL-R3 GPI-attachment signals, designated THY1-GPI(DAF) and THY1GPI(TR3). To accomplish this, a 'hinge' region was placed between mature THY1 and the foreign GPI-attachment sequences. The hinge region is based on prior publication by a group that successfully replaced the GPI-attachment signal of mouse Thy- 1 with the transmembrane domain of cluster of differentiation 8 and neural cell adhesion molecule. ${ }^{19}$ Both GPI-attachment signals consist of the $\mathrm{C}$ terminus eight residues upstream of the GPI anchorage sites of DAF and TRAIL-R3 (Figure 2a and $b$ ).

Thy- 1 is detected in RFL- 6 cells transfected with expression vectors for THY1-GPI(DAF) or THY1-GPI(TR3) as assessed by western blot using clone K117 (Figure 3a). The bands for each are the same size and in keeping with the predicted polypeptide mass difference of $\sim 1.3 \mathrm{kDa}$ relative to the band for WT Thy-1 and are correctly glycosylated. Identical results were obtained using clone 5E10 (not shown). After $24 \mathrm{~h}$ incubation with PI-PLC, cells expressing THY1-hinge-GPI (DAF) and -GPI(TR3) are completely negative for cell surface Thy- 1 as assessed by flow cytometry. This confirms that both are GPI anchored and neither GPI anchor possesses an additional palmitoyl group on the inositol. PI-PLC hydrolyzes the bond in the GPI anchor that liberates the diacylglycerol but not the palmitoyl group from the inositol. ${ }^{22}$ For both, the GPI anchor is predicted to attach 15 AA downstream of the WT attachment site of Thy-1 (Figure 2b). This is confirmed by the size of each relative to native Thy- 1 and recombinant WT Thy-1 [Figure 3a]. Thus, GPI anchors preserve the conformation required for recognition of Thy- 1 by K117 and 5E10 even when they are up to 15 AA removed from the WT attachment site. Moreover, a GPI anchor attached to Thy- 1 by 
a non-endogenous GPI anchor-attachment signal can confer a conformation recognizable by K117 and 5E10.

\section{Recombinant sThy- 1 is not Recognized by the Anti- Human Thy-1 Monoclonal Antibodies from Clones K117, 5E10, and ASO2}

Recombinant sThy-1 without a GPI anchor can be expressed in mammalian cells by introducing a stop codon downstream or in place of the codon for CYS 130 and upstream of the GPI anchor-attachment signal. ${ }^{9,18}$ Without a tether to the inner leaflet, recombinant Thy- 1 in the ER traffics to the Golgi, and then into the CM. This approach was taken in the design of recombinant sThy-1 (Figures 1a, b and 2a, b).

Two bands, approximately 25 and $20 \mathrm{kDa}$ in size, are detected in the CM of RFL- 6 transfected with expression vectors for FLAG-sTHY1 and sTHY1- $6 \times$ HIS. The slower migrating band is always at a greater intensity than the smaller band and in keeping with the predicted polypeptide mass difference, lacking a GPI anchor, and being correctly glycosylated (Figure $5 \mathrm{a}$ and $\mathrm{b}$ ). To determine if the two bands represent differentially glycosylated recombinant sThy-1, western blots were performed using sThy-1 in CM treated with PNGase F. With PNGase F treatment, the CM no longer contained the $\sim 25$ and $\sim 20 \mathrm{kDa}$ bands, but rather a single

a

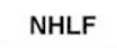

STHY1 $-6 \mathrm{X}$ HIS

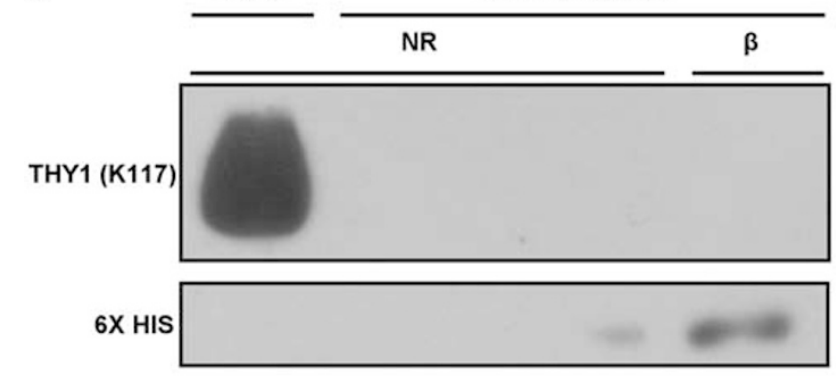

b

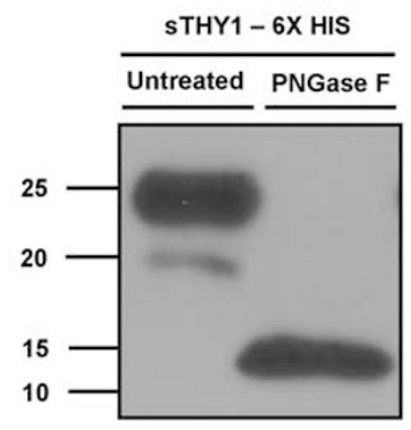

Figure 5 Characterization of soluble recombinant thymocyte differentiation antigen 1 (Thy-1) with 6-histidine $(6 \times$ HIS) epitope tag at $\mathrm{C}$ terminus. (a) Mature Thy-1 detected by western blot in the cell extract (CE) of normal human lung fibroblast (NHLF) or conditioned media (CM) containing soluble (s)THY1-6 $\times$ HIS prepared as indicated under nonreducing (NR) conditions or with $2.5 \% \beta$-mercaptoethanol $(\beta)$. After stripping the membranes of antibodies for detecting mature Thy-1, the $6 \times$ HIS epitope tag was probed for. (b) $6 \times$ HIS epitope detected by western blot in equal dilutions of untreated or peptide: $\mathrm{N}$-glycosidase $\mathrm{F}$ (PNGase F)-treated CM containing sThy-1-6 $\times$ HIS. band between 15 and $10 \mathrm{kDa}$ (Figure 5b). The predicted molecular mass of the polypeptide alone is $\sim 13.9 \mathrm{kDa}$.

$\mathrm{N}$ terminally tagged sThy- 1 was used to confirm that sufficient recombinant sThy- 1 is present in CM of transfected cells for K117, 5E10, and AS02 to be able to detect it, if it maintained the correct conformation without a complete GPI anchor. CEs from RFL-6 cells expressing FLAG-THY1and FLAG-sTHY1-containing CM were run in western blots with anti-FLAG as the primary antibody. The relative concentration of Thy- 1 in these preparations was determined in the same manner as before by assuming FLAG band intensity is directly proportional to the concentration of Thy-1. Sample preparations of CE and CM were loaded, so a greater amount of FLAG-sTHY1 was run in western blots with clone K117, 5E10, and ASO2 as the primary antibody. As with FLAG-THY1 delipidated by PI-PLC, both western blots showed a band in the lane corresponding to the cell lysates but not the CM (Figure 3b). After stripping the membranes of primary and secondary antibodies for detecting Thy-1, each was re-probed with FLAG antibody. As anticipated, there was more FLAG-sTHY1 in the lanes containing the CM (Figure $3 b$ ).

Unlike the case with anti-human Thy-1 monoclonal antibodies, recognition of the histidine epitope at the $\mathrm{C}$ terminus of sTHY1-6 $\times$ HIS requires or at least is greatly facilitated with reducing conditions (Figure 5a). Reduced and non-reduced sTHY1-6 $\times$ HIS run in adjacent lanes reveals a $\sim 25 \mathrm{kDa}$ band that spans the entire lane of the former and at the edge of the latter (Figure 5a). Even after concentrating several fold, non-reduced sTHY1- $6 \times$ HIS was never detected by western blot with K117 (data not shown).

\section{The Increased Thy-1 in the CM of Normal Human Fibroblasts Treated with Proinflammatory Cytokines, Which Coincides with a Decrease at the Cell Surface, is Insoluble}

The monoclonal antibody from clone K117 detects basal- and cytokine-induced increases in levels of Thy-1 in the CM of CCL-210. However, as demonstrated in Figure 3b, K117 does not recognize sThy-1 expressed without a GPI anchor or Thy-1 delipidated with PI-PLC. This suggests that Thy-1 detected in the CM of CCL-210 retains a complete GPI anchor and is therefore insoluble.

CCL-210 CM was partitioned into soluble and insoluble phases using the non-ionic detergent Triton X-114 to assess the solubility of Thy-1 detected in it. Insoluble and soluble phases were submitted to western blot analysis using K117 as the primary. None of the Thy- 1 detected in the CM of CCL210 was retained in the soluble phase. Rather, Thy- 1 detected in the $\mathrm{CM}$ of either cytokine stimulated or un-stimulated NHLF partitioned exclusively into the insoluble phase (Figure 6a). Moreover, the vast majority of Thy-1 detected in the pre-concentrated $\mathrm{CM}$, retained following centrifugation at $1200 \mathrm{~g}$, was removed following centrifugation at $\geq 21000 \mathrm{~g}$ (Figure 6b). 
a

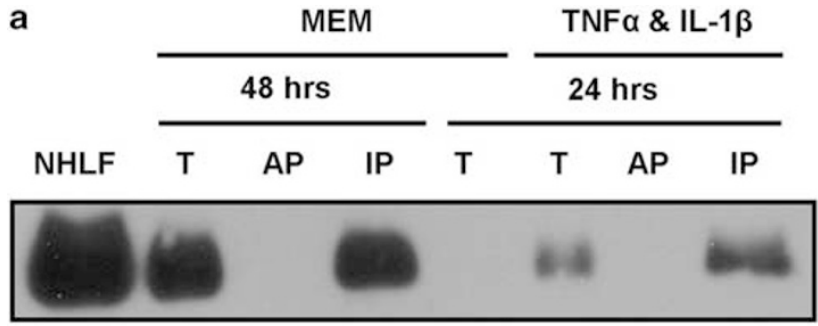

b

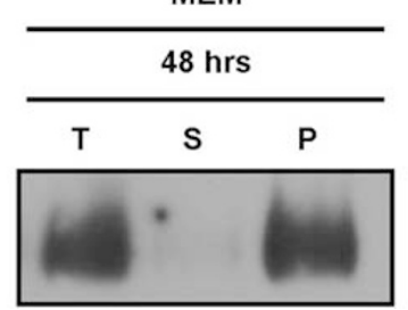

Figure 6 Thymocyte differentiation antigen 1 (Thy-1) in the conditioned media (CM) of normal human lung fibroblasts is insoluble. (a) CM was collected from CCL-210 provided either minimum essential medium (MEM) or $20 \mathrm{ng} / \mathrm{ml}$ tumor necrosis factor (TNF) $\alpha$ and interleukin (IL)- $1 \beta$ in MEM for 24 to $48 \mathrm{~h}$. Using Triton X-114, concentrated CM was partitioned into an aqueous phase (AP) and insoluble phase (IP). Mature Thy-1 detected by western blot in the cell extract (CE) of normal human lung fibroblast (NHLF) or partitioned and pre-partitioned CM by western blot using K117. (b) CM was collected from CCL-210 provided MEM $48 \mathrm{~h}$. CM was submitted to differential centrifugation of $1200 \mathrm{~g}$ and $21000 \mathrm{~g}$, and then precipitated with methanol. Mature Thy- 1 was detected in the precipitated material of total (T) before, supernatant (S) after, and pellet (P) after $21000 \mathrm{~g}$ by western blot using K117.

\section{DISCUSSION}

The monoclonal antibodies from clones K117, 5E10, and AS02 recognize human Thy-1 at the surface of cells. Thus, these antibodies recognize epitopes displayed by Thy- 1 while in a native conformation. In addition, all three detect Thy- 1 in western blots, but not under reducing conditions. This suggests that these antibodies recognize epitopes comprised of segments in the polypeptide held in close proximity by disulfide bonds. The mature Thy-1 polypeptide has four cysteine residues. As Thy- 1 is a member of the immunoglobulin superfamily, each cysteine is predicted to form a disulfide bond with one of the other under oxidizing conditions. ${ }^{21}$ The complete deglycosylation of Thy-1 does not affect the affinities these antibodies have for it. Therefore, the epitopes are likely confined to a region of the Thy- 1 polypeptide. Taken together, recognition of Thy-1 by K117, 5E10, and ASO2 requires the THY1 polypeptide be in a conformation it assumes at the surface of cell membranes.

Molecular dynamic models comparing rodent Thy- 1 inserted into a lipid monolayer, vs delipidated Thy- 1 as if by GPI-specific phospholipase D1 (GPLD1), vs without any portion of a GPI anchor, demonstrate distinct conformational differences. The conformation of rodent Thy- 1 without any portion of a GPI anchor is intermediate between the other two but closer to the GPLD1 delipidated model.
Interestingly, these same studies suggest that the glycan chain of the GPI anchor is tightly folded on itself, bringing the protein in close proximity to the cell surface. A bulk of it may even fit into a lectin-type binding site on the adjacent surface of Thy-1. ${ }^{12,23}$ The conformational differences are appreciable on the surface of Thy-1 opposite the GPI anchor; known epitopes in these areas become compromised, ${ }^{12}$ consistent with altered antibody affinity. For Thy-1- and GPI-anchored proteins, the general consensus is that delipidation induces a stable change in conformation that manifests itself in a change in antibody affinity. ${ }^{14}$ The positions of TYR residues in human Thy- 1 are better suited than those in rodent Thy-1 for using circular dichroism spectra to detect conformational changes. A discernable and stable shift in the circular dichroism spectrum of human Thy-1 occurs within an hour of its delipidation. Taken all together, antibodies that recognize human Thy-1 with a GPI anchor are predicted to have lower affinity for Thy-1 if it is delipidated.

We evaluated the relative affinity of three widely available monoclonal Thy-1 antibodies, K117, 5E10, and AS02, for the mature Thy-1 polypeptide with a complete GPI anchor, delipidated by PI-PLC, and expressed as a soluble recombinant protein by omitting the GPI-attachment signal. Of the three antibodies, AS02 was shown by another group to detect an increase of Thy-1 in the supernatant of PI-PLC-treated human fibroblast. Thy- 1 was also detected in the supernatant of untreated cells, however. ${ }^{13}$ To detect the mature Thy-1 polypeptide independent of conformation, recombinant forms were engineered to be expressed with an $\mathrm{N}$-terminal FLAG tag. Our findings demonstrate that recognition of Thy-1 by monoclonal antibodies from clones K117, 5E10, and AS02 in western blots is abolished or greatly diminished if Thy- 1 is made soluble by PI-PLC or expressed without a GPI anchorattachment signal. Remarkably, GPI anchors can mediate the conformation of Thy-1 required for recognition by K117 and 5E10 from as far away as 15 AA and attached by a nonendogenous GPI anchor-attachment signal. Moreover, detection of THY1-GPI(DAF) and -GPI(TR3) suggests that the epitopes these antibodies bind do not encompass both the polypeptide and the glycan core of the GPI anchor.

Recombinant sThy-1 has been designed in a number of different ways. In one design, three AA followed by a stop codon were introduced immediately following CYS 130 of rat Thy1.1. ${ }^{18}$ The additional AA, GGS, were included to allow it to be purified by affinity chromatography using OX-7, 18 shown to lose affinity for Thy- 1 if delipidated. ${ }^{12}$ Although our FLAG-sTHY1 has a six AA extension, it was not detected in western blots using K117, 5E10, or AS02 (Figure 3b and Supplementary Figure 1). In addition, sTHY1-6 $\times$ HIS, with a 12 AA extension, was not detected in western blots using K117. In a second design, $6 \times$ HIS followed by a stop codon were introduced immediately following LYS 129 of human Thy-1, thereby omitting CYS 130. Interestingly, this form of recombinant sThy-1 was detected in western blots by AS02. Also, it was used to establish the standard curve in a 
sandwich ELISA in which AS02 and 5E10 were the detection antibodies. ${ }^{9}$ Two potential explanations could account for why these antibodies detect this form but not ours: (1) The disulfide bond formed with CYS 130 may place a constraint at the C-terminus that sequesters elements downstream of it. This is supported by reducing conditions exposing the histidine epitope at the C terminus of sTHY1- $6 \times$ HIS in western blots. (2) Detection by AS02 and 5E10 may require a greater amount of a sThy-1 relative to the GPI-anchored form, in which case absolute values for each would not be comparable.

The integrin and syndecan- 4 binding motifs RLD and RETKK, respectively, were indentified and characterized using a recombinant hybrid of Thy-1 in which the GPIattachment signal is replaced by the Fc fragment of human IgG1. Although soluble, the Fc fragment molecular mass at $\sim 25.6 \mathrm{kDa}$ is two times the mature Thy-1 polypeptide. The relatively large size could presumably supply the constraint at the carboxyl terminus for Thy- 1 antibody recognition. Thy$1-\mathrm{Fc}$ forms a dimer ${ }^{24}$ through the $\mathrm{Fc}$ fragments, making it further unsuited as a surrogate for WT-sThy- $1 .{ }^{25}$ We tested whether commercially available Thy-1-Fc (ALX-522-091) is recognized by K117. A band just over $100 \mathrm{kDa}$ was detected, suggesting that Thy-1-Fc has a conformation more akin to GPI-anchored Thy-1 (data not shown). Although without an ability to detect Thy-1-Fc by western blot independent of its conformation, the possibility cannot be discounted that the recognition is nonspecific. It is not known whether the binding motifs RLD or RETKK are altered in the sThy-1 conformation in a manner that would influence affinity for integrins or syndecan-4. Potential affinity differences aside, the bivalent nature of Thy-1-Fc likely crosslinks cell surface receptors that native sThy-1 would not. Absent a transmembrane domain, clustering is an important mechanism by which Thy-1 signals. ${ }^{19,26}$ Thus, sThy-1-Fc most likely better approximates WT Thy- 1 at the cell surface.

There are only two known mammalian enzymes that release GPI-anchored proteins by cutting within the GPI moiety, GPLD1 ${ }^{27-29}$ and angiotensin-converting enzyme (ACE) ${ }^{30-32}$ Both have been shown to release Thy-1 from cell surfaces. ${ }^{31-33}$ ACE cleaves between the first and second mannose of the GPI moiety leaving ethanolaminephosphate-mannose. ${ }^{31,32}$ GPLD1 cleaves the inositol phosphate linkage within the GPI moiety so that the phosphate is retained by the diacylglycerol. ${ }^{14,29,34}$ Compared with the remnant GPLD1 leaves behind, ACE leaves behind one more similar in size to a small AA extension and less similar to the one PI-PLC generates. However, it is unknown if Thy-1 released by ACE is recognized by antibodies demonstrated to be deficient in recognizing other forms of sThy-1. The GPI anchor remnant, ethanolamine-phosphate-mannose, could act in a similar manner as the three AA at the C terminus of sTHY1.1-GGS.

Despite the inability to recognize sThy-1, K117 detects basal levels of Thy-1 in the CM of NHLF and the increase of

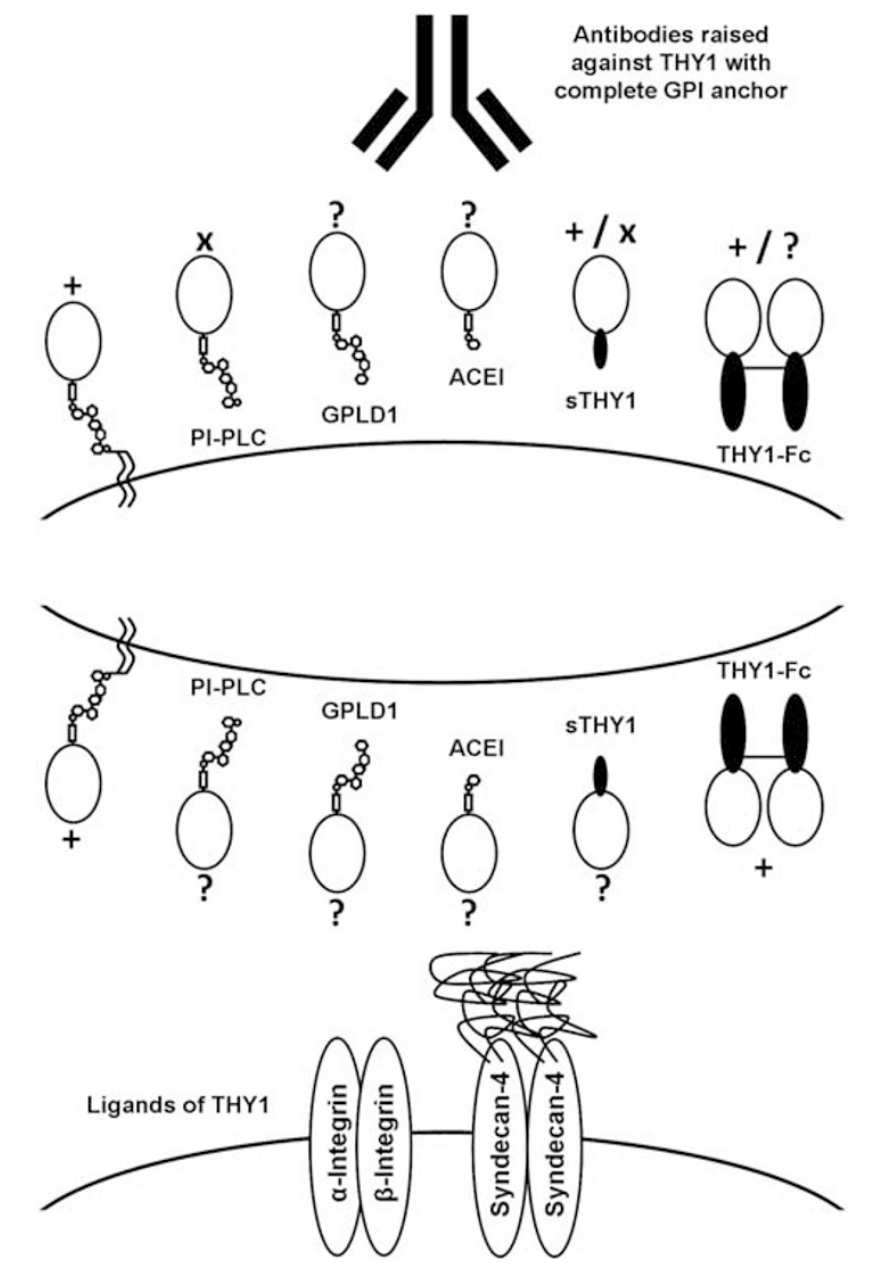

Figure 7 Diagram of glycosylphosphatidylinositol (GPI)-anchored, delipidated, and recombinant thymocyte differentiation antigen 1 (Thy-1) depicted with relative affinity to antibodies raised against GPI-anchored and known ligands of Thy-1. Delipidation induces a change in the conformation of Thy-1, causing it to lose affinity for antibodies that recognize the native conformation of THY1 with a complete GPI anchor. Recombinant soluble Thy-1, that is, expressed without a GPI-attachment signal, also has a conformation that is not recognized by these same antibodies. However, some forms of recombinant soluble Thy- 1 with either a specific amino-acid (AA) extension or possibly omission of certain AAs, that is, CYS 130, may restore affinity. This phenomenon is depicted in the upper half of the diagram, in which white ovals represent the mature Thy- 1 polypeptide and narrow black ovals are AA extensions off CYS 130. The conformation assumed by Thy-1 released from cell surfaces with GPI-specific phospholipase D1 (GPLD1) and angiotensin-converting enzyme inhibitor (ACEI) with respect to antibody affinity, is not known. Moreover, the effect conformational differences may have on the affinity for known natural ligands or whether new ones are gained is not known. All are important to consider when raising antibodies to, using a recombinant form of, or examining the function of Thy-1. IgG, immunoglobulin G; PI-PLC, phosphatidylinositol-specific phospholipase C.

Thy-1 that comes with proinflammatory cytokine treatment. These incongruous results led to experiments to assess the solubility of Thy- 1 detected in CM. As suggested, Thy- 1 detected in the CM of CCL-210 is entirely insoluble. Differential centrifugation revealed the Thy-1 to be associated 
with a rather large particle as it was cleared from the CM at speeds as low as $21000 \mathrm{~g}$. Microparticles released from cell membranes are enriched in GPI-linked proteins, and are increasingly recognized to have an important role in intracellular communication in a number of physiological processes and pathological conditions. ${ }^{35-39}$

A group recently published detection of elevated Thy-1 in the cell-free post-digestion supernatant, a by-product in processing prostate tissue for cell sorting by collagenase digestion. The presence of Thy- 1 in the supernatant was attributed to either 'cell shedding, secretion, or enzymatic release'. However, this was carried out by western blot using 5E10 as the primary antibody. ${ }^{8}$ Based on the results reported here, the Thy- 1 thus detected likely retains a complete GPI anchor. On the other hand, mass spectrometry, but not western blot, analysis of urine from patients with prostate cancer identified Thy-1; conversely, Thy-1 was not detected in the urine of post-prostatectomy patients. ${ }^{8}$ It may be that the Thy-1 in the urine in prostate cancer is a truly soluble, delipidated form.

We report that K117, 5E10, and AS02 are deficient in detecting soluble forms of Thy- 1 by western blot. Thus, there is a need to develop antibodies to Thy-1 that take into consideration the conformation of delipidated Thy-1. In addition, the conformation of recombinant forms of Thy- 1 used as controls in assays that rely on antibody recognition must be evaluated carefully. Beyond antibody recognition, the conformation of recombinant forms of Thy- 1 used to elucidate its function should also be evaluated. The aforementioned considerations are summarized and depicted in Figure 7. Furthermore, the nature of Thy-1 released by cytokines, which appears to maintain its membrane-bound conformation and may have important biological significance, needs to be evaluated further.

Supplementary Information accompanies the paper on the Laboratory Investigation website (http://www.laboratoryinvestigation.org)

\section{ACKNOWLEDGEMENTS}

We thank Drs Celia Espinoza, Kornelia Kis, Wendy Liu, and Simon Wong for their helpful suggestions and discussions. We also thank Elizabeth Boyd for all her administrative services and Dr Daniel $F$ Legler for his gift of expression vectors containing the CDNA of the GPI-attachment signals for DAF and TRAIL-R3. We acknowledge the support of the UCSD Center for AIDS Research (NIH/DAIDS, P30 AI36214, PI D Richman) Molecular Biology Core and Flow Cytometry Research Core Facilities at the VA San Diego Healthcare System. This work was also supported by National Institutes of Health Grant R01HL082818 (to JSH).

\section{DISCLOSURE/CONFLICT OF INTEREST}

The authors declare no conflict of interest.

1. Reif $A E$, Allen $J M$. Specificity of isoantisera against leukaemic and thymic lymphocytes. Nature 1963;200:1332-1333.

2. Reif $A E$, Allen $J M$. The AKR thymic antigen and its distribution in leukemias and nervous tissues. J Exp Med 1964;120:413-433.

3. Rege TA, Hagood JS. Thy-1 as a regulator of cell-cell and cell-matrix interactions in axon regeneration, apoptosis, adhesion, migration, cancer, and fibrosis. FASEB J 2006;20:1045-1054.
4. Rege TA, Hagood JS. Thy-1, a versatile modulator of signaling affecting cellular adhesion, proliferation, survival, and cytokine/growth factor responses. Biochim Biophys Acta 2006;1763:991-999.

5. Bradley JE, Ramirez G, Hagood JS. Roles and regulation of Thy-1, a context-dependent modulator of cell phenotype. Biofactors 2009;35:258-265.

6. Hagood JS, Prabhakaran P, Kumbla $P$, et al. Loss of fibroblast Thy-1 expression correlates with lung fibrogenesis. Am J Pathol 2005;167:365-379.

7. Almqvist P, Carlsson SR. Characterization of a hydrophilic form of Thy-1 purified from human cerebrospinal fluid. J Biol Chem 1988;263:12709-12715.

8. True LD, Zhang H, Ye M, et al. CD90/THY1 is overexpressed in prostate cancer-associated fibroblasts and could serve as a cancer biomarker. Mod Pathol 2010;23:1346-1356.

9. Saalbach A, Wetzig T, Haustein UF, et al. Detection of human soluble Thy- 1 in serum by ELISA. Fibroblasts and activated endothelial cells are a possible source of soluble Thy- 1 in serum. Cell Tissue Res 1999;298:307-315.

10. Zhou Y, Hagood JS, Lu B, et al. Thy-1-integrin alphav beta5 interactions inhibit lung fibroblast contraction-induced latent transforming growth factor-beta1 activation and myofibroblast differentiation. J Biol Chem 2010;285:22382-22393.

11. Kukulansky $\mathrm{T}$, Abramovitch $\mathrm{S}$, Hollander $\mathrm{N}$. Cleavage of the glycosylphosphatidylinositol anchor affects the reactivity of thy-1 with antibodies. J Immunol 1999;162:5993-5997.

12. Barboni E, Rivero BP, George AJ, et al. The glycophosphatidylinositol anchor affects the conformation of Thy-1 protein. J Cell Sci 1995;108(Part 2):487-497.

13. Saalbach A, Kraft R, Herrmann K, et al. The monoclonal antibody ASO2 recognizes a protein on human fibroblasts being highly homologous to Thy-1. Arch Dermatol Res 1998;290:360-366.

14. Bütikofer $\mathrm{P}$, Malherbe $\mathrm{T}$, Boschung $\mathrm{M}$, et al. GPI-anchored proteins: now you see 'em, now you don't. FASEB J 2001;15:545-548.

15. Kozak M. An analysis of $5^{\prime}$-noncoding sequences from 699 vertebrate messenger RNAs. Nucleic Acids Res 1987;15:8125-8148.

16. Einhauer A, Jungbauer A. The FLAG peptide, a versatile fusion tag for the purification of recombinant proteins. J Biochem Biophys Methods 2001;49:455-465.

17. Hopp TP, Prickett $\mathrm{KS}$, Price VL, et al. A short polypeptide marker sequence useful for recombinant protein identification and purification. Nat Biotechnol 1988;6:1204-1210.

18. Devasahayam M, Catalino PD, Rudd PM, et al. The glycan processing and site occupancy of recombinant Thy-1 is markedly affected by the presence of a glycosylphosphatidylinositol anchor. Glycobiology 1999;9:1381-1387.

19. Tiveron MC, Nosten-Bertrand $M$, Jani $H$, et al. The mode of anchorage to the cell surface determines both the function and the membrane location of Thy-1 glycoprotein. J Cell Sci 1994;107(Part 7):1783-1796.

20. Hoessli D, Bron C, Pink JR. T-lymphocyte differentiation is accompanied by increase in sialic acid content of Thy- 1 antigen. Nature 1980;283:576-578.

21. Perkins SJ, Williams AF, Rademacher TW, et al. The Thy-1 glycoprotein: a three-dimensional model. Trends Biochem Sci 1988;13:302-303.

22. Roberts WL, Myher JJ, Kuksis A, et al. Lipid analysis of the glycoinositol phospholipid membrane anchor of human erythrocyte acetylcholinesterase. Palmitoylation of inositol results in resistance to phosphatidylinositolspecific phospholipase C. J Biol Chem 1988;263:18766-18775.

23. Rademacher TW, Edge CJ, Dwek RA. Dropping anchor with the lipophosphoglycans. Curr Biol 1991;1:41-42.

24. Leyton $L$, Schneider P, Labra CV, et al. Thy-1 binds to integrin $\beta 3$ on astrocytes and triggers formation of focal contact sites. Curr Biol 2001;11:1028-1038.

25. Avalos AM, Valdivia AD, Muñoz N, et al. Neuronal Thy-1 induces astrocyte adhesion by engaging syndecan-4 in a cooperative interaction with alphavbeta3 integrin that activates PKCalpha and RhoA. J Cell Sci 2009;122(Part 19):3462-3471.

26. Mayor S, Rothberg KG, Maxfield FR. Sequestration of GPI-anchored proteins in caveolae triggered by cross-linking. Science 1994;264:1948-1951.

27. Low MG, Prasad AR. A phospholipase D specific for the phosphatidylinositol anchor of cell-surface proteins is abundant in plasma. Proc Natl Acad Sci USA 1988;85:980-984. 
28. Davitz MA, Hom J, Schenkman S. Purification of a glycosylphosphatidylinositol-specific phospholipase D from human plasma. J Biol Chem 1989;264:13760-13764.

29. Scallon BJ, Fung WJ, Tsang TC, et al. Primary structure and functiona activity of a phosphatidylinositol-glycan-specific phospholipase D. Science 1991;252:446-448.

30. Kondoh G, Watanabe $\mathrm{H}$, Tashima $\mathrm{Y}$, et al. Testicular angiotensinconverting enzyme with different glycan modification: characterization on glycosylphosphatidylinositol-anchored protein releasing and dipeptidase activities. J Biochem 2009;145:115-121.

31. Kondoh G, Tojo H, Nakatani $Y$, et al. Angiotensin-converting enzyme is a GPI-anchored protein releasing factor crucial for fertilization. Nat Med 2005;11:160-166.

32. Mayor S. ACEing GPI release. Nat Struct Mol Biol 2005;12:107-108.

33. Bergman AS, Carlsson SR. Saponin-induced release of cell-surfaceanchored Thy-1 by serum glycosylphosphatidylinositol-specific phospholipase D. Biochem J 1994;298(Part 3):661-668.
34. Metz CN, Brunner G, Choi-Muira NH, et al. Release of GPI-anchored membrane proteins by a cell-associated GPI-specific phospholipase D. EMBO J 1994;13:1741-1751.

35. Tetta C, Bruno S, Fonsato V, et al. The role of microvesicles in tissue repair. Organogenesis 2011;7:105-115.

36. Muralidharan-Chari V, Clancy JW, Sedgwick A, et al. Microvesicles: mediators of extracellular communication during cancer progression. J Cell Sci 2010;123:1603-1611.

37. Butikofer P, Kuypers FA, Xu CM, et al. Enrichment of two glycosylphosphatidylinositol-anchored proteins, acetylcholinesterase and decay accelerating factor, in vesicles released from human red blood cells. Blood 1989;74:1481-1485.

38. Kim H-S, Choi D-Y, Yun SJ, et al. Proteomic analysis of microvesicles derived from human mesenchymal stem cells. J Proteome Res 2011;11:839-849.

39. Anderson HC, Mulhall D, Garimella R. Role of extracellular membrane vesicles in the pathogenesis of various diseases, including cancer, renal diseases, atherosclerosis, and arthritis. Lab Invest 2010;90:1549-1557. 